\title{
Post-Processing of Electrocardiographic Imaging Signals to Identify Atrial Fibrillation Drivers
}

\author{
Rubén Molero ${ }^{1}$, Andreu M Climent ${ }^{1}$, María S Guillem ${ }^{1}$ \\ ${ }^{1}$ ITACA Institute, Universitat Politècnica de València, Valencia, Spain
}

\begin{abstract}
Phase mapping of inverse-computed electrograms (ECGI) is used for rotor activity identification to guide ablation procedures in atrial fibrillation $(A F)$ patients. This study aims at identifying the post-processing settings that best allow tracking meaningful rotors.

ECGI signals of $24 \mathrm{AF}$ patients prior to pulmonary vein isolation (PVI) were recorded. Patients were divided according to their 6 months outcome (sinus vs. no sinus). Rotor metrics were compared using 3 types of processing: none, a narrow band-pass filtering centered at the highest dominant frequency (NB HDF), and sinusoidal recomposition filtering (SRC). Different thresholds for rotor duration were also compared (0.5, 1, and 1.5 turns).

Differences in the absolute error of rotor metrics between sinus and no sinus patients were best identified with raw ECGI signals than filtered ECGI signals. In contrast, rotor duration threshold had little effect on the statistical differentiation between groups.

Filtering of AF ECGI signals does not improve rotor identification to predict PVI outcome.
\end{abstract}

\section{Introduction}

Electrocardiographic imaging (ECGI) is used to characterize the cardiac electrical activity by using body surface electrocardiograms and the anatomy of the patient and guide ablation procedures in patients with atrial fibrillation (AF). However, the accuracy of ECGI maps to detect the presence of sources maintaining AF has not been extensively validated because it is unfeasible to record the actual electrograms of human patients during AF with a sufficient resolution, since current mapping techniques have a spatial resolution that is larger than the size of the atrial wavefronts. We have shown that there is some correlation between the complexity metrics of propagation patterns of intracardiac and ECGI mapping in AF patients [1]. However, this match between intracardiac and ECGI AF maps is not perfect and it is difficult to determine how much this is due to a lack of accuracy of ECGI because of a poor resolution of the inverse problem, to a poor accuracy of intracardiac mapping, with a limited spatial resolution and areas that cannot be mapped by basket catheters, or to a poor post-processing for rotor identification.

Rotors are typically identified by phase mapping after computing the Hilbert transform of ECGI signals [2]. This transform set values from $-\pi$ to $+\pi$ to each signal sample and is used to define reentrant cardiac activity. Singularity Points (SP) are defined as a propagation pattern that pivots a point in the cardiac surface oscillating in the phase ranges of $[-\pi,+\pi]$. While a rotor should complete at least 1 turn in the phase map to be considered a rotor, changes in the propagation direction may appear as SPs lasting for less than 1 turn. In addition, far field contributions incorrectly avoided by solving the inverse problem do result in discontinuities in rotor detection and thus resulting in real rotors lasting for less than 1 turn [3]. We have previously reported that more than 1 turn should be required to achieve a sufficient specificity in identifying rotors by using computer modeled AF [4], but this has not been validated with human data, for which the loss of sensitivity by imposing an increased amount of turns to the presence of rotors, and its resulting loss in sensitivity may decrease the ability to identify rotors at all.

The potential loss of sensitivity in the detection of rotors can be improved by filtering the ECG signals and thus reduce the contribution of the electrical propagations taking place at different frequencies other than the rotor [3]. However, in our simulation studies we have shown that this increased sensitivity has a trade-off in a lack of specificity [4]. Alternative post-processing techniques could be better suited for this, such as sinusoidal recomposition (SRC) [5]. However, this has not been validated as of today.

The objective of the present study is to identify which are the best post-processing techniques to identify atrial rotors, namely filtering of ECGI signals and the amount to turns required to SPs to be defined as rotors. We will base our selection criteria in maximizing the differences in the variation among time of rotor metrics with a favorable and unfavorable outcome after pulmonary vein isolation (PVI), under the assumption that the underlying electrical characteristics of these two groups of patients should be different and identifiable by ECGI.

\section{Material and methods}

\subsection{Data acquisition and processing}

Body Surface Potential Mapping (BSPM) signals were recorded using 57 channels from 24 patients (18 females 
and 6 males; $61.8 \pm 14.3$ years old) with atrial fibrillation prior to pulmonary vein isolation (PVI). Patients were classified into two groups depending on the success of PVI at 6 months after ablation (patients with sinus rhythm at 6 months, $\mathrm{N}=13$ vs. patients with atrial arrhythmias, including atrial fibrillation, atrial tachycardia and atrial flutter, $\mathrm{N}=11$ ). Torso anatomy and the electrode location were obtained using photogrammetry techniques. MRI/CT scan images were recorded to obtain atria's anatomy that was segmented using ITK-SNAP software [6]. Torso and atria anatomies were co-registered using the torso reference from MRI/CT images.

BSPM signals of 4 seconds of duration were band-pass filtered between 2 and $45 \mathrm{~Hz}$ to eliminate noise, and the QRST segment then canceled lead by lead by PCA approach [7]. Inverse computed electrograms (ECGI) of each signal were calculated by zero-order Tikhonov regularization and L-curve optimization [8].

\subsection{ECGI filtering and Rotor identification}

Two different filters were applied to ECGI signals: a narrow band-pass filter at the highest dominant frequency $(\mathrm{HDF} \pm 0.5 \mathrm{~Hz})$, and a filter based on a sinusoidal recomposition [5]. Singularity Points of the raw and filtered ECGI signals were calculated using Hilbert's transform to compute the phase signal [4]. Phase singularity points (SP) were defined as a propagation pattern that pivots a point in epicardium oscillating in the phase ranges of $[-\pi,+\pi]$ and rotors were defined as singularity points connected in time and space. Three different thresholds were established to consider an SP as valid rotor: $0.5,1$, and 1.5 turns.

\subsection{Rotor metrics and statistical analysis}

Different metrics to quantify rotor behavior in $\mathrm{AF}$ patients were calculated: the number of unconnected rotors per second, mean time with rotors (\%) and mean rotor duration (s). The absolute error between two metrics of each patient was calculated and non-paired t-student test of each error was performed to compare the patients with different outcomes 6 months after PVI (sinus vs. no sinus).

\section{Results}

\subsection{Filtering and SP detection}

The largest amount of unconnected rotors was found for raw ECGI signals than for both SRC or HDF filtered signals for duration thresholds of 0.5 and 1 turns (136 \pm 104 and $46 \pm 38$ respectively), and no big difference for 1.5 turns, as it can be observed in Fig. 1. Mean time with rotors was not significantly different among filtering strategies for 0.5 turns (no filter: $95 \pm 6.8 \%$, SRC: $94 \pm 6.9 \%$ and NB at HDF: $96 \pm 3.3 \%$ ), but was significantly decreased with an increasing threshold of the number of turns required for both raw signals and SRC filtered (threshold 1.5 turns, no filter: $65 \pm 31 \%$, SRC: $67 \pm 26 \%$ ). Mean time with rotors, however, was not significantly reduced for NB $\mathrm{HDF}$ filtered signals with time restrictions for the rotors detected and remained at $96 \pm 3.8 \%$ and $95 \pm 4.5 \%$ for 1 and 1.5 turns, respectively. Mean rotor duration increases at higher turn thresholds (no filter at 0.5 turns: $0.07 \pm 0.02$ s, and for 1.5 turn: $0.14 \pm 0.09 \mathrm{~s}$ ). When post-processing filters were applied, mean rotor duration increased (1.5 turns SRC: $0.21 \pm 0.09 \mathrm{~s}, \mathrm{NB}$ HDF: $0.48 \pm 0.15 \mathrm{~s}$ ).

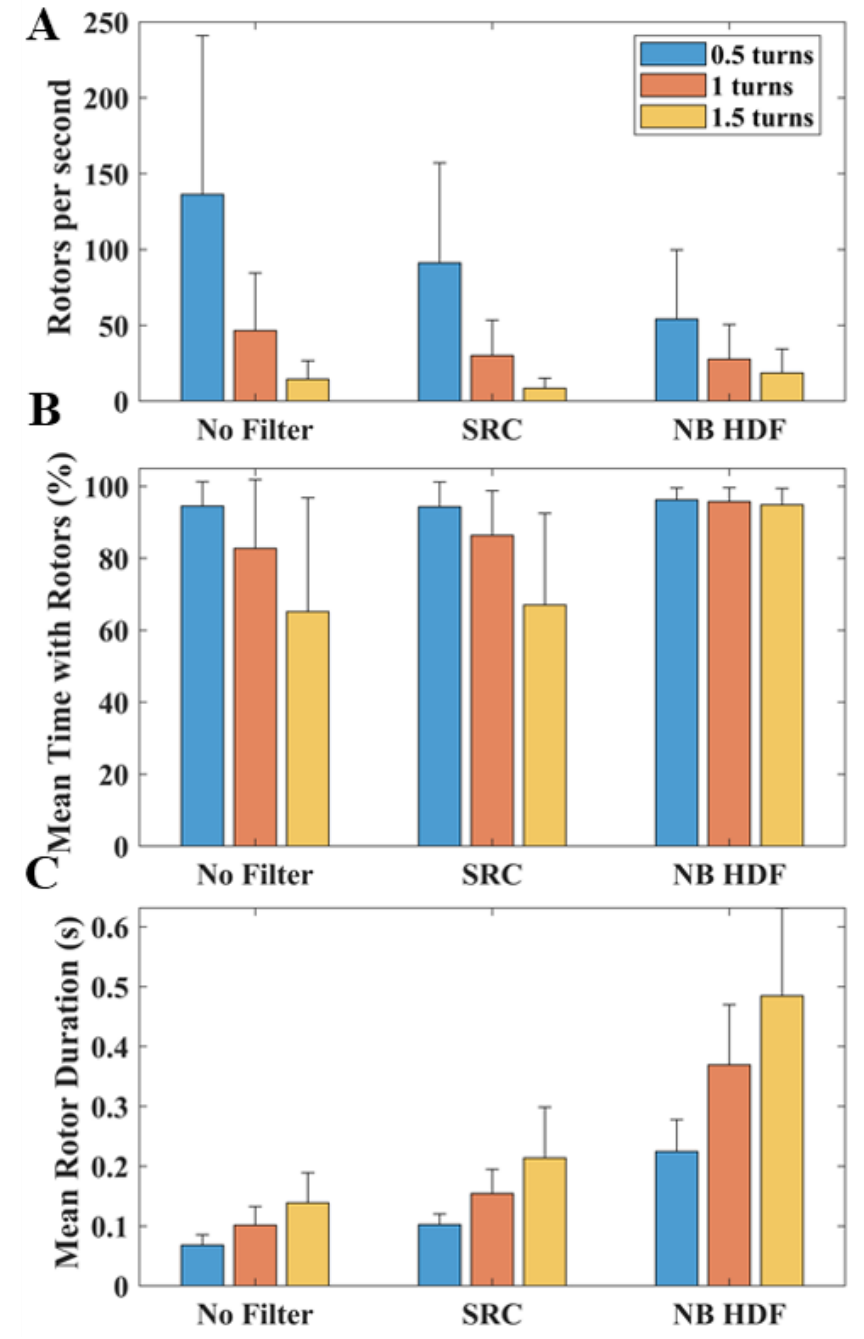

Figure 1. Rotors per second (A), mean time with rotors (\%) (B) and mean rotor duration (s) (C) calculated for ECGI with no filter, Kuklik filter and a narrow band-pass filter in the high dominant frequency using three SP thresholds: $0.5,1$ and 1.5 turns.

An example of the effect of the different postprocessing techniques on the identification of rotors is 
given in Fig.2, where rotor histogram maps of the same ECGI signal for the nine scenarios are depicted. All the maps clearly show the SP presence and at similar locations. Narrow band-pass maps (Fig. 2 G, H, I) show a larger amount of SP compared with the other two types of filters. For the case of 1.5 turns threshold, the presence of SP decreases but the area where the rotors are is well delimited.

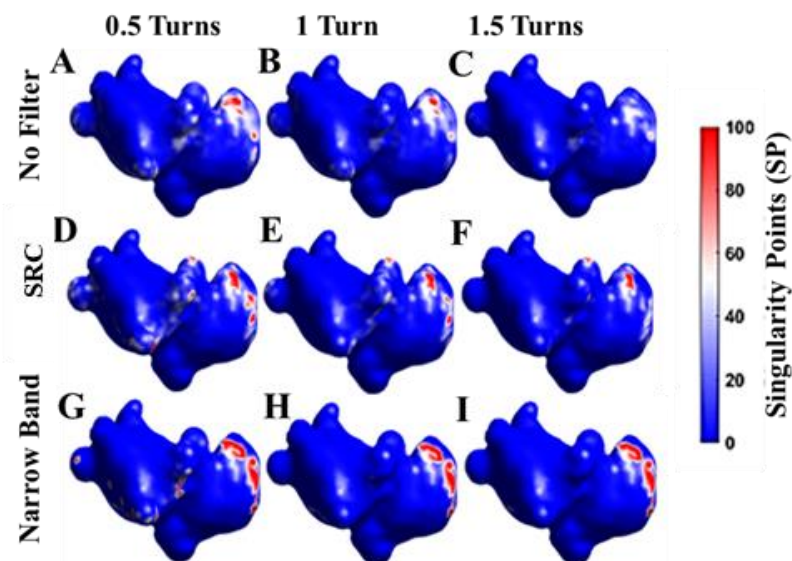

Figure 2. Singularity points presence maps of the ECGI signals with different filters and SP detection thresholds.

\subsection{Patient classification comparison}

Fig. 3 shows mean values and standard deviation diagrams of the absolute error between two quantified rotor metrics for both the sinus and no sinus groups 6 months after PVI and Table 1 shows the p-values of unpaired t-tests. Patients with successful PVI at 6 months showed a lower variation in time of the number of rotors per second than patients with bad PVI results independently on the filtering strategy or amounts of turns required (Fig. 3A-D). Differences in mean time with rotors were found to be higher for patients with unsuccessful PVI (Fig. 3B-E) for both raw signals and SRC filtered, with any temporal threshold applied. However, differences are more significant for a 0.5 rotations threshold, with lower pvalues. Narrow band-pass filtering, however, does not show differences among groups for the differences in mean time with rotors. Finally, error in mean rotor duration did not show significant differences between patient groups for filtered signals. (Fig. 3C-F) However, differences were significant for filtered ECGI signals for 1 turn case and close to significance for the rest of threshold showing a clear difference compared to the significance of the filtered alternatives.

\section{Discussion}

This study presents a comparison of different ECGI signal processing methods and different criteria for rotor detection. This study also provides results of how patients with different AF prognosis after PVI respond after the proposed filtering and SP evaluation scenarios.

Previous studies that made use of simulated atrial signals [4] showed that NB HDF filtering increases the sensitivity in the detection of rotors at the expense of reducing the specificity when applied to ECGI signals.
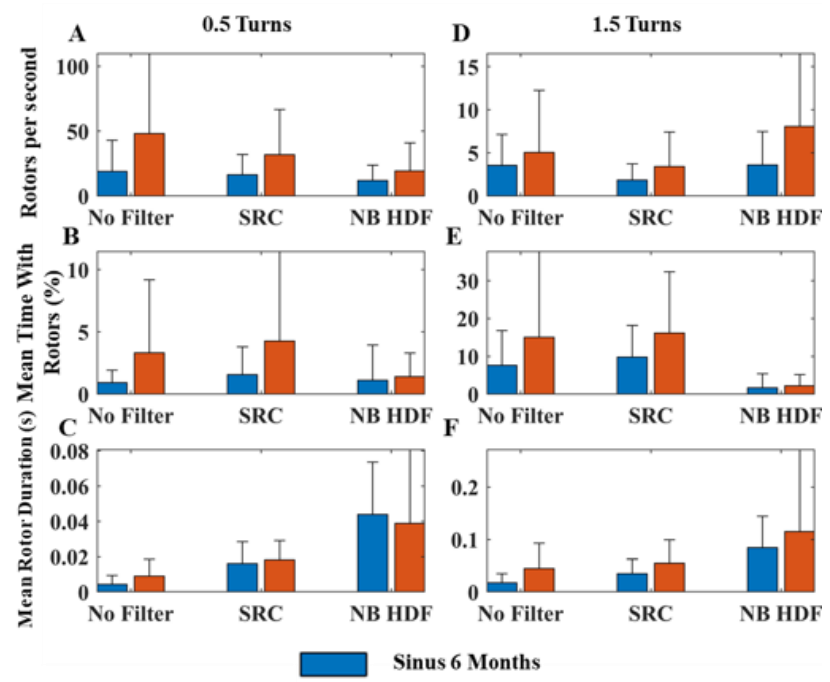

Figure 3. Mean absolute error between two measurements of rotors per second, mean time with rotors and mean rotor duration comparison between patients with good and bad outcome of pulmonary vein isolation at six moths of ECGI signals with no filter, SRC filter and a narrow band-pass filter at highest dominant frequency using 0.5 turns for SP detection (A, B and C) and 1.5 turns (D, E, F).

However, this observation was not validated with real patient data for which the trade-off between sensitivity and specificity may have been different. Even though it is not possible to know in a real case scenario which of our detected rotors are real and which rotors are artefactual, our study reports that having this increased sensitivity will not allow to find differences in rotor metrics related to the outcome of patients after PVI, while these differences can be observed in raw ECGI signals.

Applying SRC of the ECGI signal previous SP detection doesn't show to change quantitatively the metric results compared to the raw signal, however, it hinders the distinction between patients as compared to the raw signal, as p-values are higher. SRC [5] alleviates the effect of confounding features on the phase of the signal but does not improve on raw ECGI signals.

SP detection using different turn thresholds shows evidences that with a more restrictive detection value, the singularities detected decrease. Besides, no differences between SP detection criteria and major differences between groups of patients were observed. Only the filtering of the ECGI signals affects to characteristic metrics of a delimited sample of patients with the same prognosis. 


\begin{tabular}{c|c|c|c|c|c|c|c|c|c}
\hline & \multicolumn{3}{|c|}{0.5 Turns } & \multicolumn{3}{c|}{ 1 Turn } & \multicolumn{3}{c}{1.5 Turns } \\
\hline Metric & ECGI & SRC & NB HDF & ECGI & SRC & NB HDF & ECGI & SRC & NB HDF \\
\hline Rotors per second & 0.13 & 0.16 & 0.30 & 0.13 & 0.28 & 0.18 & 0.52 & 0.22 & 0.10 \\
\hline Mean Time with Rotors (\%) & 0.16 & 0.21 & 0.77 & 0.18 & 0.34 & 0.15 & 0.29 & 0.23 & 0.72 \\
\hline Mean Rotor Duration (s) & 0.13 & 0.67 & 0.74 & 0.03 & 0.97 & 0.84 & 0.07 & 0.20 & 0.52 \\
\hline
\end{tabular}

Table 1: P-value of unpaired t-test results of the absolute difference of two measurements between patients with sinus rhythm and no sinus rhythm 6 months after PVI for ECGI signals with different filters and thresholds for SP detection

\section{Conclusion}

Aggressive filtering strategies of atrial ECGI signals are not necessary to identify relevant rotor features. Rotor metrics based on raw ECGI signals allows for a better differentiation of patients with different prognosis after pulmonary vein isolation.

The duration threshold for the rotational activity found has not shown to be critical for identifying relevant rotors. While a more severe restriction in the amount of turns required does decrease the number of detected rotors, the differences between patient groups are consistent among different thresholds applied.

\section{Acknowledgments}

Supported in part by: Instituto de Salud Carlos III FEDER (Fondo Europeo de Desarrollo Regional PI17/01106) and EIT Health (Activity code 19600). EIT Health is supported by EIT, a body of the European Union.

\section{References}

[1]M. Rodrigo et al., "Noninvasive assessment of complexity of atrial fibrillation, correlation with contact mapping and impact of ablation", Circ. Arrhy. Electrophysiol., vol. 13, No. 03, Feb. 2020.

[2]S. Zlochiver et al. "Rotor meandering contributes to irregularity in electrograms during atrial fibrillation", Heart Rhythm, vol. 5, pp. 846-854, Jun. 2008.
[3]M.Rodrigo et al., "Body surface localization of left and right atrial high-frequency rotors in atrial fibrillation patients: A clinical-computational study", Heart Rhythm, vol.11., pp-15841591, May 2014.

[4]M. Rodrigo et al., "Technical considerations on phase mapping for identification of atrial reentrant activity in direct and inverse computed electrograms", Circ. Arrhy. Electrophisiol., vol. 10, Sept. 2017.

[5]P. Kuklik et al., "Reconstruction of instantaneous phase of unipolar atrial contact electrogram using a concept of sinusoidal recomposition and Hilbert transform", IEEE Trans. Biomed. Engin, vol 62, pp. 296-302, Jan. 2015.

[6] P. Yushkevich et al., "Continuous medial representation for anatomical structures", IEEE Trans. Med. Imaging, vol. 25, no. 12, pp. 1547-64, Dec. 2006.

[7]F. Castells et al. "Estimation of atrial fibrillatory wave from single-lead atrial fibrillation electrocardiograms using principal component analysis concepts", Med. Biol. Eng. Comput., vol 43, pp. 557-560, Oct. 2005.

[8]M. Rodrigo et al., "Highest dominant frequency and rotor positions are robust markers of driver location during noninvasive mapping of atrial fibrillation: a computational study," Heart Rhythm, vol. 14, no. 8, pp. 1224-1233, Aug. 2017.

Address for correspondence:

Rubén Molero Alabau.

ITACA. Edificio 8G acceso B. Universitat Politécnica de València. Camino de Vera s/n. 46022 Valencia, Spain. rumoal1@itaca.upv.es 